\title{
Проверка модели обращенно-фазовой ВЭЖХ, разработанной на базе теории обобщенных зарядов, и ее применение для оптимизации подвижной фазы
}

\author{
Пономарев Ф.В., Долгоносов А.М. \\ Институт геохимии и аналитической химии им. В.И. Вернадского Российской академии наук, Москва
}

Поступила в редакцию 30.01.2018 г.

В статье применен основанный на теории обобщенных зарядов новый подход к описанию удерживания в ВЭЖХ. Адекватность теоретической модели продемонстрирована на примере изократической системы обращенно-фазовой ВЭЖХ с нафталином в качестве аналита. Показана возможность выбора многокомпонентной подвижной фазы, имеющей заданную элюотропную силу. Разработана программа для оптимизации состава подвижной фазы по экономическому критерию.

Ключевые слова: теория обобщенных зарядов, обращенно-фазовая хроматография, фактор удерживания, элюотропная сила, оптимизация многокомпонентной подвижной фазы.

\section{Verification of the reversed-phase HPLC model developed on the basis of the generalized charges theory and its application for mobile phase optimization}

\author{
Ponomarev P.V., Dolgonosov A.M. \\ V.I. Vernadsky Institute of Geochemistry and Analytical Chemistry, RAS, Moscow
}

Nowadays there is a problem of theoretical model suitable for describing the HPLC process and predicting its most important parameters. For the liquid chromatography processes there are special mechanisms of adsoption, «hydrophobic interaction», and competitive sorption. In general, the sorption equilibrium description in liquid chromatography combines the features of the processes of physical adsorption from the rarefied gas medium and strictly competitive ion-exchange. Moreover, solvation processes play an important role in liquid phase. Current theoretical approaches often defining one of the several problems with the others consideration omitting not allowed to create universal and sufficiently convenient model for the wide field of all HPLC considered stationary phases and analytes. In the vast majority of modern and classical concepts including the famous models of Snyder, Soczewinsky and Scott-Kuchera there is a large number of empirical parameters.

Previously from the simplified model for weak eluents the phenomenological theory describing the particular cases of the known approaches and having a small number of transferable parameters was developed for the describing of chromatography. The generalized charges theory is used for the transferable parameters evaluation.

Formulas for the non-empirical calculation of the basic model parameters are given. The small but undoubted experimental material showing the satisfactory accuracy of a priori theoretical calculations based on the developed model is found in the literature. The theoretical model is applied to the reversed-phase HPLC isocratic system with water-acetonitrile eluent and naphthalene as an analyte. The possibility of optimization of the multicomponent mobile phase with given eluotropic force according to the economic criterion is shown in the paper. It has been held by using the program which was specially designed for research purpose in Python environment. 
Application of the new composition under the experiment conditions would make possible to achieve almost $40 \%$ of economy. The calculation algorithm presented in the article will be a basis for the further HPLC modeling both for describing the behavior of analytes and for choosing the optimal conditions for their separation.

Keywords: generalized charges theory, reversed-phase chromatography, retention factor, eluotropic force, multicomponent mobile phase optimization

\section{Введение}

Одной из основных проблем теории жидкостной хроматографии является отсутствие универсальной модели, подходящей для описания данного процесса и предсказания важнейших его параметров, таких как времена или факторы удерживания анализируемых соединений. В последнее время написано множество монографий и научных статей по данной тематике [1-3], однако в подавляющем большинстве работ в основе описываемых концепций лежит большое число эмпирических параметров, тогда как основной задачей представляется создание отлаженной методологии на основе априорных предсказаний, что повлечет за собой сокращение экспериментальной работы. Стоит отметить, что для наиболее полного описания жидкостной хроматографии необходимо учитывать вклад полярных взаимодействий, в том числе электростатических сил и водородных связей.

Из ряда классических теорий можно выделить подходы Снайдера и Сочевинского $[4,5]$ и близкие им, которые рассматривают термодинамику конкурентной сорбции без подробного описания механизма распределения и пренебрегают эффектами физической адсорбции на свободной поверхности; в то время как подход Скотта-Кучеры [6] описывает конкретные модели адсорбции и сольватации. Форма записи основных уравнений в этих подходах имеет характерные отличия. При всем при том там используются эмпирические коэффициенты, поэтому не приходится говорить о фундаментальности выражений и их предсказательной силе, трудно выявить взаимосвязь рассчитываемых параметров со структурой молекул. Основное уравнение модели Снайдера:

$$
\lg k_{i}=a_{0}+a_{1} v+a_{2} v^{2}
$$

где $k_{i}$ - фактор удерживания, $a_{0}, a_{1}, a_{2}$ - эмпирические константы, $v$ - объемная доля модификатора. Основное уравнение модели Скотта-Кучеры:

$$
\frac{1}{k_{i}}=A+B v
$$

где $A, B$ - эмпирические константы.

Для создания теоретической модели следует исходить из физических уравнений для энергии взаимодействий в многокомпонентной системе. Эти взаимодействия описываются сложным механизмом физической и конкурентной адсорбции. Подобный же механизм встречается и при сольватации молекул как в нормальнофазовой, так и в обращенно-фазовой ВЭЖХ. В результате общие вклады в данный процесс аналогичны ионному обмену с конкурентной сорбцией и газовой хроматографии с физической адсорбцией. Последний вклад проявляется в минорной степени, однако его роль важна для определения связи параметров процессов в ВЭЖХ со структурой молекул [7-12].

В распространенном случае применения в ВЭЖХ подвижной фазы (ПФ), включающей в себя как органические модификаторы, так и ионные (обычно минеральные) компоненты для создания нужной среды, коэффициент распределения определяется суммой ионообменного и адсорбционного вкладов: 


$$
\Gamma_{i}=\frac{K_{i j}{ }^{z_{i}}}{\varphi_{e x}^{z_{i}}}+\frac{\alpha_{i j} \Gamma_{0 i}}{\left(\varphi_{n s}^{\prime}\right)^{n_{i j}}}
$$

где $\Gamma_{\mathrm{i}}, \Gamma_{0 \mathrm{i}}$ - искомая и нормированная константы Генри (коэффициенты распределения); $K_{i}^{z_{i}}$ - константа ионного обмена; $\alpha_{i j}$ - параметр конкурентной сорбции; $n_{i j}$ - параметр молекулярной площадки; $z_{i}$ - заряд иона; $\varphi_{e x}^{z_{i}}$ и $\varphi_{n s}^{\prime}$ - элюотропные силы ионного и неполярного вкладов в адсорбцию.

Первое слагаемое уравнения (3) связано с ионообменной силой $\varphi_{e x}$, тогда как второе слагаемое $\varphi_{n s}^{\prime}$ представляет собой вклад конкурентных взаимодействий для обращенно-фазовой хроматографии. Непосредственно из формулы (3) для неполярной части, в предположении работы полной поверхности неподвижной фазы следует основное уравнение для элюотропной силы многокомпонентной подвижной фазы в обращенно-фазовой жидкостной хроматографии:

$$
\sum\left(\varphi^{\prime}\right)^{-n_{j l}} \alpha_{j l} \kappa_{j} v_{j}=1,
$$

где $\varphi^{\prime}$ - элюотропная сила; $\kappa_{j}$ - нормированная константа Генри; $v_{j}$ - объемная доля компонента; кроме индекса $j$ компонентов подвижной фазы для удобства расчетов используется понятие опорного компонента (l-компонента) [13-16].

В данной работе приводятся первые изыскания на пути к универсальной комплексной теории моделирования ВЭЖХ, включающие описание хроматографической системы с помощью теории обобщенных зарядов $[17,18]$, разработанной нашей научной группой, а также создание программного комплекса для оптимизации состава элюирующей смеси с промежуточным сравнением работы модели и программы с экспериментальными данными. В качестве объектного аналита был выбран нафталин, который является достаточно сложным полиароматическим соединением и среди других подобных молекул обладает большой величиной времени удерживания в обращенно-фазовой ВЭЖХ, что является благоприятным из соображений проверки точности расчета.

\section{Теоретическая часть}

Развиваемая модель базируется на уравнениях (5)-(18), приведенных в таблице $1[17,18]$. С ее помощью осуществляли априорный расчет сорбционных величин для исследуемых объектов.

Таблица 1. Формулы для расчета характеристик по теории обобщенных зарядов

\begin{tabular}{|c|c|c|}
\hline Величина & \multicolumn{2}{|c|}{ Выражение } \\
\hline 1 \\
$\begin{array}{c}\text { Электронный объ- } \\
\text { ем (аддитивная ха- } \\
\text { рактеристика числа } \\
\text { валентных электро- } \\
\text { нов молекулы) }\end{array}$ & $V_{i}=N_{\sigma i}+N_{\pi i} \sqrt{2}$ & (5) \\
\hline Обобщенный заряд & $Q_{i}=\left(V_{i}-\pi_{d i} \sqrt{2}\right)^{\frac{3}{4}}+\left(\pi_{d i} \sqrt{2}\right)^{\frac{3}{4}}=\left(N_{\sigma i}+N_{\pi i} \sqrt{2}-\pi_{d i} \sqrt{2}\right)^{\frac{3}{4}}+\left(\pi_{d i} \sqrt{2}\right)^{\frac{3}{4}}$ & (6) \\
\hline $\begin{array}{c}\text { Параметр молеку- } \\
\text { лярной площадки }\end{array}$ & $n_{i j}=\frac{Q_{i}}{Q_{j}}=\frac{\left(N_{\sigma i}+N_{\pi i} \sqrt{2}-\pi_{d i} \sqrt{2}\right)^{\frac{3}{4}}+\left(\pi_{d i} \sqrt{2}\right)^{\frac{3}{4}}}{\left(N_{\sigma j}+N_{\pi j} \sqrt{2}-\pi_{d j} \sqrt{2}\right)^{\frac{3}{4}}+\left(\pi_{d j} \sqrt{2}\right)^{\frac{3}{4}}}$ & (7) \\
\hline
\end{tabular}

Пономарев и др. / Сорбционные и хроматографические процессы. 2018. Т. 18. № 1 


\begin{tabular}{|c|c|c|}
\hline 1 & 2 & \\
\hline $\begin{array}{c}\text { Характеристика } \\
\text { жесткости молеку- } \\
\text { лы }\end{array}$ & $g_{i}=\left(\sum \mu_{i} \cdot V_{i}\right)^{-\frac{3}{4}}=\left(\sum \frac{m_{\text {іатома }}}{M_{\text {молекульь }}} \cdot V_{i}\right)^{-\frac{3}{4}}$ & (8) \\
\hline $\begin{array}{c}\text { Константа моди- } \\
\text { фикатора }\end{array}$ & $G_{j}=D-\frac{g_{j}}{2} \cdot Q_{j}-\frac{u_{b m}}{R T} \cdot Q_{j}^{2}$ & (9) \\
\hline $\begin{array}{l}\text { Параметр конку- } \\
\text { рентной сорбции }\end{array}$ & $\ln \alpha_{i j}=\left(n_{i j}-1\right) G_{j}+\frac{1}{2} \ln \frac{m_{i}}{n_{i j} m_{j}}$ & (10) \\
\hline $\begin{array}{c}\text { Энергетический } \\
\text { фактор молекулы в } \\
\text { поле адсорбента } \\
\end{array}$ & $B_{i}=-\frac{U_{0 A}}{R T}-\frac{g_{i}}{2}$ & (11) \\
\hline $\begin{array}{l}\text { Нормированная } \\
\text { константа Генри }\end{array}$ & $\kappa_{i}=\exp \left(B_{i} Q_{i}+D\right)$ & (12) \\
\hline $\begin{array}{l}\text { Относительное } \\
\text { удерживание }\end{array}$ & $\ln y=-n_{i j} \ln \left[1+\left(\kappa_{j}-1\right) v_{j}\right]+\theta\left[v_{j} \ln \alpha_{i j}-\kappa_{j}^{-1}\left(1-v_{j}\right)\left(1-\alpha_{i j}\right)\right]$ & (13) \\
\hline $\begin{array}{l}\text { Коэффициент рас- } \\
\text { пределения в сис- } \\
\text { теме без модифи- } \\
\text { катора }\end{array}$ & $\Gamma_{0 i}=\kappa_{i} \frac{a_{1 i}}{c_{1 i}}$ & (14) \\
\hline $\begin{array}{l}\text { Коэффициент рас- } \\
\text { пределения }\end{array}$ & $\Gamma_{i}=\Gamma_{0 i} \cdot y$ & (15) \\
\hline $\begin{array}{l}\text { Фактор удержива- } \\
\text { ния }\end{array}$ & $k_{i}=\frac{\Gamma_{i}}{\varepsilon}$ & (16) \\
\hline
\end{tabular}

Электронный объем, являющийся линейной комбинацией числа валентных $\sigma$ $\left(N_{\sigma}\right)$ и $\pi$-электронов $\left(N_{\pi}\right)$ молекулы, служит мерой электронной плотности (5). Процедура расчета аддитивного показателя обобщенного заряда для нескольких жестких фрагментов молекул, объединенных нежесткими связями (6), может учитывать мезомерные электроны ( $\pi_{d} \sqrt{2}$, где $\pi_{d}$ - половина числа мезомерных электронов), тогда число остальных электронов равно $\left(V-\pi_{d} \sqrt{2}\right)$. После этого получают параметр молекулярной площадки, связанный со стехиометрией обмена адсорбата (iкомпонент) и модификатора ( $j$-компонент) (7).

С помощью нахождения параметра жесткости (8), учитывающего вклад каждого атома в электронный объем молекулы, с использованием системного значения, определяемого из условий для растворителя $(D=-0.33)$, рассчитывается константа модификатора (9). Для оценки энергии межмолекулярных сил при заданной температуре $T$ наряду с обобщенным зарядом используется теоретическая константа $u_{b m}=88.54$ Дж/моль [17]. Показатель конкурентной сорбции служит мерой обмена двух различных компонентов подвижной фазы (10). Энергетический фактор молекулы (11), в выражение для которого входит сорбционный потенциал поверхности адсорбента ( $U_{0 A}=-1.67$ кДж/моль для поверхности алкилированного силикагеля) и температура, позволяет рассчитать нормированную константу Генри (коэффициент распределения) (12).

Параметр относительного удерживания $y$ для аналита при использовании ПФ с объемной долей модификатора $v_{j}$ и долей занятой поверхности его молекулами $\theta$ вычисляется по формуле (13). Коэффициент отношения сорбционной емкости к предельной концентрации адсорбата при удельной площади поверхности адсорбента

Пономарев и др. / Сорбционные и хроматографические процессы. 2018. Т. 18. № 1 
$300 \mathrm{~m}^{2} / \mathrm{cm}^{3}$ и плотности чистого нафталина 1,14 г $/ \mathrm{cm}^{3}$ составляет $\frac{a_{1 i}}{c_{1 i}} \approx 0.084$ для нафталина и позволяет перейти сначала к нормированному коэффициенту распределения в системе без модификатора (14), а затем и к его искомому значению в реальной системе (15). Фактор удерживания связан с коэффициентом распределения и величиной порозности укладки $\varepsilon$ (обычно равной 0.33 ) по формуле (16).

\section{Эксперимент}

Экспериментальные данные были получены из технического отчета компании «Sigma-Aldrich» [19]. Для анализа был взят результат эксперимента в изократическом режиме при следующих условиях: колонка С18, 150x3 мм, 5 мкм; подвижная фаза: вода:ацетонитрил=20:80 (об.\%); скорость потока: $0.4 \mathrm{~cm}^{3} /$ мин (общий объем $1.5 \mathrm{~cm}^{3}$ за один проход); температура: $35^{\circ} \mathrm{C}(308.15 \mathrm{~K})$; инжекция 1.5 мкл; давление: 61 бар; $N_{\text {нафталина }} \sim 1000$ (число теоретических тарелок, показатель эффективности); $\Gamma_{\text {нафталина }}=1.78$. Порядок элюирования анализируемых соединений: урацил, фенол, ацетофенон, бензол, толуол, нафталин (рис. 1).

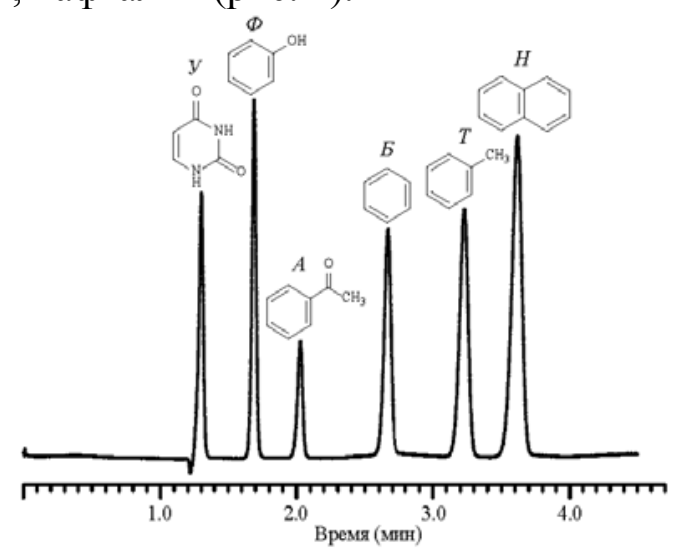

Рис. 1. Изучаемая экспериментальная хроматограмма, где У - урацил, Ф - фенол, А - ацетофенон, Б - бензол, Т - толуол, Н - нафталин

Приведенные параметры экспериментальной хроматографической системы были использованы для изучения корелляции теоретических и экспериментальных данных по нафталину.

С помощью компьютерной программы оптимизации состава, написанной в среде языка Python [20] и работающей на основе модели вложенных циклов, получали минимальное значение стоимости многокомпонентного элюента, состоявшего из четырех популярных растворителей с учетом цен за 1 литр ( $\left.p_{i}\right)$ из каталога «ThermoFischer Scientific» [21], приведенных в таблице 2.

Таблица 2. Растворители, используемые в ходе оптимизации состава элюента, и их цены

\begin{tabular}{|c|c|c|c|c|}
\hline № п/п & Название растворителя & $\begin{array}{c}\text { Объемная } \\
\text { доля, \% }\end{array}$ & Рациональная формула & $\begin{array}{c}\text { Цена за 1 литр } \\
p_{i}, \text { USD }\end{array}$ \\
\hline 1 & Ацетонитрил & $\geq 99.9$ & $\mathrm{CH}_{3} \mathrm{CN}$ & 152.10 \\
\hline 2 & Метанол & $\geq 99.9$ & $\mathrm{CH}_{3} \mathrm{OH}$ & 56.19 \\
\hline 3 & Тетрагидрофуран & $\geq 99.9$ & $\mathrm{C}_{4} \mathrm{H}_{8} \mathrm{O}$ & 135.00 \\
\hline 4 & Изопропанол & $\geq 99.9$ & $\left(\mathrm{CH}_{3}\right)_{2} \mathrm{CHOH}$ & 76.38 \\
\hline
\end{tabular}

Пономарев и др. / Сорбционные и хроматографические процессы. 2018. Т. 18. № 1 
Стоимость элюирующего состава находили путем перемножения представленных выше цен $p_{i}$ на объемные доли компонентов:

$$
P=p_{1} v_{1}+p_{2} v_{2}+p_{3} v_{3}+p_{4} v_{4}
$$

Условный алгоритм программы, выводившей в результате минимальную стоимость элюента и соответствующий ей вектор состава, представлен на рис. 2.

Молекулярные массы и число электронов $N_{\sigma}, N_{\pi}$ и $\pi_{d}$, рассчитанные на основании элементного и структурного представления исследуемых молекул, обобщены в таблице 3.

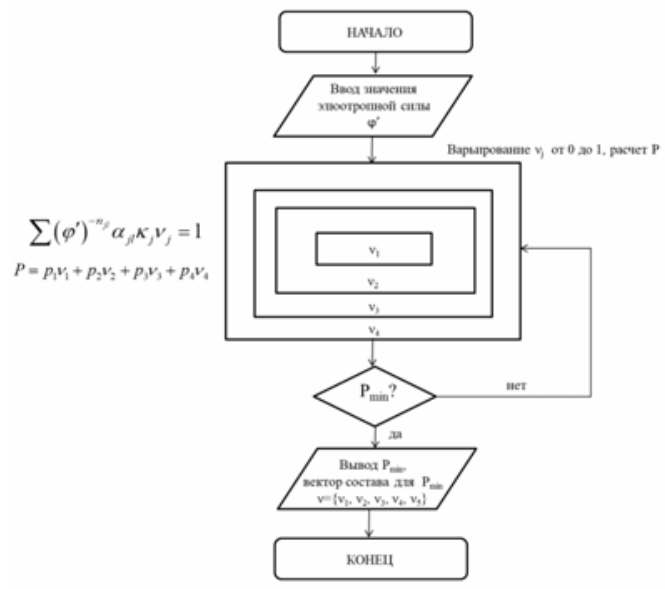

Рис. 2. Алгоритм программы для оптимизации состава многокомпонентного элюента

Таблица 3. Молекулярные массы и число электронов молекул, учитываемых в расчетах теории обобщенных зарядов

\begin{tabular}{|c|c|c|c|c|}
\hline $\begin{array}{c}\text { Наименование мо- } \\
\text { лекулы }\end{array}$ & $\begin{array}{c}\text { Мол. мас- } \\
\text { са, Да }\end{array}$ & $\begin{array}{c}\text { Число валент- } \\
\text { ных } \sigma- \\
\text { электронов мо- } \\
\text { лекулы, } N_{\sigma}\end{array}$ & $\begin{array}{c}\text { Число } \pi- \\
\text { электронов мо- } \\
\text { лекулы, } N_{\pi}\end{array}$ & $\begin{array}{c}\text { Половина мезо- } \\
\text { мерных } \pi- \\
\text { электронов мо- } \\
\text { лекулы, } \pi_{d}\end{array}$ \\
\hline Ацетонитрил & 41.05 & 10 & 4 & 0 \\
\hline Метанол & 32.04 & 10 & 0 & 0 \\
\hline Тетрагидрофуран & 72.11 & 26 & 0 & 0 \\
\hline Изопропанол & 60.10 & 22 & 0 & 5 \\
\hline Нафталин & 128.17 & 38 & 10 & \\
\hline
\end{tabular}

\section{Обсуждение результатов}

Для оценки возможности количественных предсказаний в развиваемой теории были осуществлены расчеты сорбционных величин для всех веществ, перечисленных в экспериментальной части. Ниже приводится пример расчета для ацетонитрила. Электронный объем, обобщенный заряд и показатель молекулярной площадки для ацетонитрила рассчитывались по уравнениям (5) -(7):

$$
\begin{gathered}
Q_{\mathrm{CH}_{3} \mathrm{CN}}=(10+4 \sqrt{2})^{\frac{3}{4}}=7.87 \\
Q_{\mathrm{CH}_{3} \mathrm{OH}}=10^{\frac{3}{4}}=5.62
\end{gathered}
$$




$$
n_{\mathrm{CH}_{3} \mathrm{CN} / \mathrm{CH}_{3} \mathrm{OH}}=\frac{(10+4 \sqrt{2})^{\frac{3}{4}}}{10^{\frac{3}{4}}}=1.40
$$

Параметр конкурентной сорбции ацетонитрила относительно метанола с предварительным нахождением показателя жесткости при задании температуры 308.15 К (8) и после вычисления константы модификатора (9) был посчитан по уравнению (10):

$$
\begin{gathered}
g_{\mathrm{CH}_{3} \mathrm{CN}}=\left(\frac{41.05}{4 \cdot 12.01+3 \cdot 1.01+(2+2 \sqrt{2}) \cdot 12.01+(1+2 \sqrt{2}) \cdot 14.01}\right)^{\frac{3}{4}}=0.36 \\
G_{\mathrm{CH}_{3} \mathrm{CN}}=-0.33-\frac{0.36}{2} \cdot 7.87+\frac{88.54}{8.314 \cdot 308.15} \cdot 7.87^{2}=0.39 \\
\alpha_{\mathrm{CH}_{3} \mathrm{CN} / \mathrm{CH} \mathrm{H}_{3} \mathrm{OH}}=\exp \left((1.40-1) \cdot(-0.62)+\frac{1}{2} \cdot \ln \left(\frac{41.05}{1.40 \cdot 32.04}\right)\right)=0.75
\end{gathered}
$$

Расчет нормированной константы Генри осуществлялся по формулам (11)(12) с использованием ранее найденных значений

$$
\begin{gathered}
B_{\mathrm{CH}_{3} \mathrm{CN}}=-\frac{(-1670)}{8.314 \cdot 308.15}-\frac{0.36}{2}=0.47 \\
\kappa_{\mathrm{CH}_{3} \mathrm{CN}}=\exp (0.47 \cdot 7.87+(-0.33))=29.05
\end{gathered}
$$

Аналогично в тех же условиях были рассчитаны величины для трех других растворителей, их параметры представлены в таблице 4.

Таблица 4. Величины, рассчитанные с помощью теории обобщенных зарядов для растворителей многокомпонентного элюента по отношению к метанолу $(l)$ при температуре $308.15 \mathrm{~K}$

\begin{tabular}{|c|c|c|c|c|c|c|c|}
\hline Молекула, $j$ & $Q_{j}$ & $n_{j l}$ & $g_{j}$ & $G_{j}$ & $\alpha_{j l}$ & $B_{j}$ & $\kappa_{j}$ \\
\hline Ацетонитрил & 7.87 & 1.40 & 0.36 & 0.39 & 0.75 & 0.47 & 29.05 \\
\hline Метанол & 5.62 & 1.00 & 0.49 & -0.62 & 1.00 & 0.41 & 7.20 \\
\hline Тетрагидрофуран & 11.51 & 2.05 & 0.42 & 1.83 & 0.55 & 0.44 & 113.80 \\
\hline Изопропанол & 10.16 & 1.81 & 0.43 & 1.05 & 0.62 & 0.44 & 62.83 \\
\hline
\end{tabular}

Задача определения фактора удерживания аналита была рассмотрена для нафталина. Расчет его характеристик был проведен по отношению к элюенту с модификатором ацетонитрилом:

$$
\begin{gathered}
Q_{\mathrm{C}_{10} \mathrm{H}_{8}}=(38+10 \sqrt{2}-5 \sqrt{2})^{\frac{3}{4}}+(5 \sqrt{2})^{\frac{3}{4}}=21.73 \\
n_{\mathrm{C}_{10} \mathrm{H}_{8} / \mathrm{CH}_{3} \mathrm{CN}}=\frac{(38+10 \sqrt{2}-5 \sqrt{2})^{\frac{3}{4}}+(5 \sqrt{2})^{\frac{3}{4}}}{(10+4 \sqrt{2})^{\frac{3}{4}}}=2.76 \\
\alpha_{\mathrm{C}_{10} \mathrm{H}_{8} / \mathrm{CH}_{3} \mathrm{CN}}=\exp \left((2.76-1) \cdot 0.39+\frac{1}{2} \cdot \ln \left(\frac{128.17}{2.76 \cdot 41.05}\right)\right)=2.11,
\end{gathered}
$$

где $i$-компонент $-\mathrm{C}_{10} \mathrm{H}_{8}$ (аналит); $j$-компонент $-\mathrm{CH}_{3} \mathrm{CN}$ (модификатор);

$$
\begin{gathered}
g_{C_{10} H_{8}}=\left(\frac{128.17}{10 \cdot 3 \cdot 12.01+10 \cdot 1 \cdot \sqrt{2} \cdot 12.01+8 \cdot 1 \cdot 1.01}\right)^{\frac{3}{4}}=0.34 \\
B_{C_{10} H_{8}}=-\frac{(-1670)}{8.314 \cdot 308.15}-\frac{0.34}{2}=0.48
\end{gathered}
$$


Ключевым этапом было получение относительного удерживания аналита (13) с учетом эффектов взаимной сольватации с элюентом в приближении полностью занятой поверхности адсорбента $\theta=1$ :

$$
\ln y=-2.76 \cdot \ln [1+0.8 \cdot(29.05-1)]+0.8 \cdot \ln (2.11)-\frac{(1-0.8) \cdot(1-2.11)}{29.05}=-8.10
$$

Константа Генри для нафталина в системе ОФЖХ с удельной поверхностью $300 \mathrm{~m}^{2} / \mathrm{cm}^{3}(14)$ равна:

$$
\Gamma_{0 C_{10} H_{8}}=0.084 \cdot \exp (0.48 \cdot 21.73+(-0.33))=2046
$$

Выражение для искомого коэффициента распределения (15) можно записать как:

$$
\Gamma_{C_{10} H_{8}}=2046 \cdot \exp (-8.10)=0.62
$$

Тогда, предполагая среднюю порозность укладки в ОФЖХ-колонке С18алкилированного силикагеля $\varepsilon=0.33$, найдем значение фактора удерживания (16):

$$
k_{\text {meop }}=\frac{0.62}{0.33}=1.88
$$

Из данных [19], приведенных в экспериментальной части по удерживанию нафталина, следует:

$$
k_{\text {эксп }}=1.78
$$

Расхождение между двумя результатами равно:

$$
\Delta k=|1.78-1.88|=0.11
$$

Относительная ошибка расчета составила $6.2 \%$.

Значение относительной ошибки около 6\% является приемлемым для рассматриваемой новой модели, построенной на основе теории обобщенных зарядов. Теперь рассмотрим задачу оптимизации состава ПФ. В качестве критерия оптимальности выберем стоимость 1 л ПФ, необходимую для заданного разделения. Примем результаты разделения, представленные на рис. 1 в экспериментальной части за цель. Таким образом, требуется заменить ПФ из 80\%-ного водного раствора ацетонитрила составом с той же элюотропной силой, но с наименьшей стоимостью. Определим элюотропную силу ацетонитрильного элюента по формуле (17), выведенной из выражения (4), где в качестве опорного компонента выбран метанол:

$$
\varphi^{\prime}=\left(\alpha_{\mathrm{CH}_{3} \mathrm{CN} / \mathrm{CH}_{3} \mathrm{OH}} \cdot \kappa_{\mathrm{CH}_{3} \mathrm{CN}} \cdot v_{\mathrm{CH}_{3} \mathrm{CN}}\right)^{\frac{1}{n_{\mathrm{CH}_{3} \mathrm{CN} / \mathrm{CH}} \mathrm{OH}}},
$$

При подстановке численных параметров получено значение элюотропной силы $\varphi^{\prime}=7.70$. С помощью разработанной программы проведена многомерная оптимизация состава элюента, результатом которой являлся состав заданной элюотропной силы, обладавший минимальной стоимостью. Значения стоимости получались после ступенчатого варьирования объемной доли каждого компонента с последующим умножением на его цену за 1 дм $^{3}$ (19) из каталога «ThermoFischer Scientific» [21] и осуществлением финальной минимизации. График работы программы для четырех широко используемых в жидкостной хроматографии растворителей и при вычисленных выше значениях приведен на рис. 3.

Введя найденное выше значение элюотропной силы в программу для расчета минимальной стоимости и вектора состава элюентной смеси было получено, что наиболее оптимальным является элюент стоимостью 74.05 USD за литр, состоящий из 19 (об.\%) ацетонитрила, 79 (об.\%) метанола, 1 (об.\%) изопропанола и 1 (об.\%) воды. Экономическая эффективность использования элюента составила:

$$
\Delta P=0.8 \cdot 152.10-74.05=47.63(U S D) \text {, т.е. почти } 40 \% .
$$




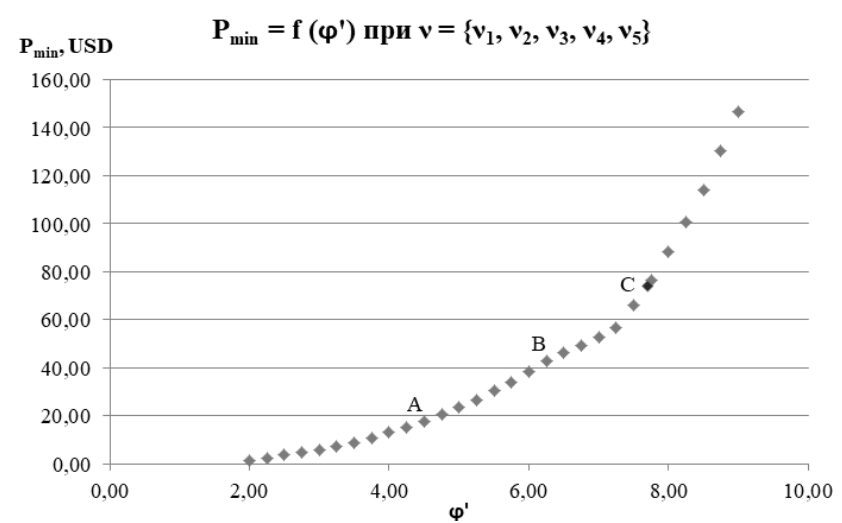

Рис. 3. Зависимость стоимости состава элюента из четырех выбранных растворителей от элюирующей силы от $\varphi^{\prime}=2.00$ до $\varphi^{\prime}=9.00$ с шагом 0.25 в скобках указан вектор состава, компонентами которого являются: \# 1 Ацетонитрил $\left(\mathrm{CH}_{3} \mathrm{CN}\right)$; \# $2 \mathrm{Me}-$ танол $\left[\mathrm{CH}_{3} \mathrm{OH}\right]$; \# 3 Тетрагидрофуран $\left[\mathrm{C}_{4} \mathrm{H}_{8} \mathrm{O}\right]$; \# 4 Изопропанол $\left[\left(\mathrm{CH}_{3}\right)_{2} \mathrm{CHOH}\right]$; \# 5 Вода $\left[\mathrm{H}_{2} \mathrm{O}\right]$. Координаты для точки А: $\varphi^{\prime}=4.50, v=\{0.00,0.00,0.00,0.23,0.77\}, \mathrm{P}_{\min }=17.57 \mathrm{USD} ;$ точки B: $\varphi^{\prime}=6.25$, $v=\{0,00,0.14,0.00,0.46,0.40\}, P_{\min }=43.00$ USD; искомой точки C: $\varphi^{\prime}=7.70, v=\{0.19,0.79,0.00,0.01$, $0,01\}, \mathrm{P}_{\min }=74.05$ USD

Таким образом, использование нового состава в условиях рассмотренного эксперимента позволило бы добиться почти 40\%-ной экономии денежных средств.

\section{Заключение}

В данной статье приведен пример априорного расчета удерживания аналита по молекулярной структуре аналита и подвижной фазы и теоретического подхода к оптимизации состава подвижной фазы для обращенно-фазовой жидкостной хроматографии, что в дальнейшем послужит созданию универсальной модели процесса жидкостной хроматографии на основании фундаментальных величин и соотношений для прогноза поведения веществ по структуре молекул и оптимизации элюента.

Авторы статьи выражают глубокую благодарность

д.ф.-м.н., А.Г. Прудковскому за ценные консультации при программировании.

Работа выполнена при финансовой поддержке РФФИ (грант 18-03-00382a).

\section{Список литературы}

1. Ammons R.L.M. Mathematical Control Theory for Liquid Chromatography. Dissertation (Ph.D.). California Institute of Technology, $1992,388 \mathrm{p}$.

2. Gu T., Liu M., .Cheng K-S.C., Ramaswamy S., Wang C.A. // Chemical Engineering Science. 2011. Vol. 66. No 15. pp. 3531-3539.

3. Snyder L.R., Dolan J.W. High-performance gradient elution. The Practical Application of the Linear-Solvent-Strength Model. Hoboken. New Jersey. John Wiley \& Sons. Inc. 2007. 461 p.
4. Snyder L.R. Principles of adsorption chromatography: The separation of nonionic organic compounds. Madison Avenue, New York, Marcel Dekker. Inc. 1968. 413 p.

5. Soczewinski E. // Anal. Chem. 1969. Vol. 41. No 1. pp. 179-182.

6. Scott R.P.W., Kucera P. // J. Chromatogr. A. 1975. Vol. 112. pp. 425-442.

7. Gu T., Zheng Y. // Separation and Purification Technology. 1999. Vol. 15. No 1. pp. 4158. 
8. Baeza-Baeza J.J., Ortiz-Bolsico C, TorresLapasió J.R, García-Álvarez-Coque M.C. // J. Chromatogr. A. 2013. Vol. 1284. pp. 28-35.

9. Fanigliulo A., Cabooter D., Bellazzi G., Alliery B. et al. // J. Chromatogr. A. 2011. Vol. 1218. No 21. pp. 3351-3359.

10. Wu D., Lucy C.A. // J. Chromatogr. A. 2017. Vol. 1516. pp. 64-70.

11. Chen Y., Brill G.M., Benz N.J., Leanna M.R. et al. // J. Chromatogr. B. 2007. Vol. 858. No 1. pp. 106-117.

12. Davies N.H., Euerby M.R., McCalley D.V. // J. Chromatogr. B. 2008. Vol. 1178. No 1.pp. 71-78.

13. Долгоносов А.М. Неспецифическая селективность в проблеме моделирования высокоэффективной хроматографии. M. URSS. 2013. $256 \mathrm{c}$.

14. Долгоносов А.М. // Сорбиионные $u$ хроматографические проиессы. 2011. Т. 11. № 4. C. 435-448.

15. Долгоносов А.М. // Сорбиионные и хроматографические проиессы. 2011. Т. 11. № 4. C.449-458.

16. Долгоносов А.М. // Сорбиионные и хроматографические проиессы. 2013. Т. 13. № 2. C.141-149.

17. Долгоносов А.М. Модель электронного газа и теория обобщенных зарядов для описания межатомных сил и адсорбиии. М. URSS. 2009. $176 \mathrm{c}$.

18. Долгоносов А.М. // Известия Академии наук. Серия химическая. 2016. Т. 65. № 4. C. $952-963$.

\section{References}

1. Ammons R.L.M. Mathematical Control Theory for Liquid Chromatography. Dissertation (Ph.D.). California Institute of Technology, 1992, $388 \mathrm{p}$.

2. Gu T., Liu M., .Cheng K-S.C., Ramaswamy S., Wang C.A., Chemical Engineering Science, 2011, Vol. 66, No 15, pp. 3531-3539.

3. Snyder L.R., Dolan J.W. High-performance gradient elution. The Practical Application of the Linear-Solvent-Strength Model. Hoboken, New Jersey, John Wiley \& Sons, Inc., 2007, $461 \mathrm{p}$.

4. Snyder L.R. Principles of adsorption chromatography: The separation of nonionic organic compounds. Madison Avenue, New York, Marcel Dekker, Inc., 1968, 413 p.
19. Henry R.A. Keys to High Speed HPLC with Isocratic Mobile Phases. Technical Report. Supelco, Division of Sigma-Aldrich. 595 North Harrison Road, Bellefonte. PA 16823. 2010. Режим доступа: https://www.sigmaaldrich.com/content/dam/sig maaldrich/docs/Supelco/General Information/1/t409 227.pdf (дата обращения 28.01.2018).

20. Python (programming language): Python 3.6.4 Release. Режим доступа: https://www.python.org/downloads/release/pyth on-364/; $\quad$ https://pypi.python.org/pypi; https://docs.python.org/3/installing/ (дата обращения 28.01.2018).

21. ThermoFisher Scientific Catalog. Режим доступа: https://www.fishersci.com/shop /products/acetonitrile-hplc-fisher-chemical-

7/a998sk1? searchHijack=true\&searchTerm $=$ A9 98SK1\&search Type $=$ RAPID\&matchedCatNo= A998SK1; https://www.fishersci.com/shop/ products/methanol-hplc-fisher-chemical6/a452sk1? searchHijack =true\&searchTerm $=\mathrm{A} 4$ 52SK1\&search Type $=$ RAPID\&matchedCat $\mathrm{No}=$ A452SK1; https://www.fishersci.com/shop/ products/tetrahydrofuran-hplc-fisher-chemical5/t425sk1? searchHijack=true\&search Term $=\mathrm{T} 42$ $5 \mathrm{SK} 1 \&$ search Type $=$ RAPID\&matchedCat $\mathrm{No}=\mathrm{T}$ 425SK1; https://www.fishersci.com /shop/products/2-propanol-hplc-fisherchemical-5/a451sk1?searchHijack=true $\&$ searchTerm $=$ A451 SK1\&searchType $=$ RAPID \&matchedCatNo=A451SK1 (дата обращения 28.01.2018).

5. Soczewinski E., Anal. Chem., 1969, Vol. 41, No 1, pp. 179-182.

6. Scott R.P.W., Kucera P., J. Chromatogr. A, 1975, Vol. 112, pp. 425-442.

7. Gu T., Zheng Y., Separation and Purification Technology, 1999, Vol. 15, No 1, pp. 4158.

8. Baeza-Baeza J.J., Ortiz-Bolsico C, TorresLapasió J.R, García-Álvarez-Coque M.C., J. Chromatogr. A, 2013, Vol. 1284, pp. 28-35.

9. Fanigliulo A., Cabooter D., Bellazzi G., Alliery B. et al., J. Chromatogr. A, 2011, Vol. 1218, No 21, pp. 3351-3359.

10. Wu D., Lucy C.A., J. Chromatogr. A, 2017, Vol. 1516, pp. 64-70. 
11. Chen Y., Brill G.M., Benz N.J., Leanna M.R. et al., J. Chromatogr. B, 2007. Vol. 858, No 1, pp. 106-117.

12.Davies N.H., Euerby M.R., McCalley D.V., J. Chromatogr. B, 2008, Vol. 1178, No 1, pp. 71-78.

13. Dolgonosov A.M. Nespetsificheskaya selektivnost' $\mathrm{v}$ probleme modelirovaniya vysokoeffektivnoi khromatografii. M., URSS, 2013, 256 p. (Rus).

14. Dolgonosov A.M., Sorbtsionnye i khromatograficheskie protsessy, 2011, Vol. 11, No 4, pp. 435-448.

15.Dolgonosov A.M., Sorbtsionnye i khromatograficheskie protsessy, 2011, Vol. 11, No 4, pp. 449-458.

16. Dolgonosov A.M., Sorbtsionnye i khromatograficheskie protsessy, 2013, Vol. 13, No 2, pp. 141-149.

17. Dolgonosov A.M. Model' elektronnogo gaza i teoriya obobshchennykh zaryadov dlya opisaniya mezhatomnykh sil i adsorbtsii. M., URSS, 2009, 176 p. (Rus).

18. Dolgonosov A.M., Izvestiya Akademii nauk. Seriya khimicheskaya, 2016, Vol. 65, No 4, pp. 952-963.

19. Henry R.A. Keys to High Speed HPLC with Isocratic Mobile Phases Technical Report. Supelco, Division of Sigma-Aldrich. 595 North Harrison Road, Bellefonte, PA 16823, 2010. Available at: https://www.sigmaaldrich.com/ content/dam/sigma-aldrich/docs

Пономарев Филипп Валерьевич - аспирант, лаборатория сорбционных методов, ГЕОХИ РАН, Москва

Долгоносов Анатолий Михайлович - д.х.н., ведущий научный сотрудник, лаборатория сорбционных методов, ГЕОХИ РАН, профессор кафедры химии, Университет «Дубна», Москва, еmail: amdolgo@mail.ru
/Supelco/General_Information/1/t409227.pdf (accessed 28 January 2018).

20. Python (programming language): Python 3.6.4 Release. Режим доступа: https://www.python.org/downloads/release/pyth on-364/; $\quad$ https://pypi.python.org/pypi; https://docs.python.org/3/installing/ (accessed 28 January 2018).

21. ThermoFisher Scientific Catalog. Available at:

https://www.fishersci.com/shop/products/aceton itrile-hplc-fisher-chemical-

7/a998sk1? searchHijack $=$ true \&search Term $=$ A9

98SK1\&search Type $=$ RAPID\&matchedCatNo= A998SK1;

https://www.fishersci.com/shop/products/metha nol-hplc-fisher-chemical-

6/a452sk1? searchHijack $=$ true\&searchTerm $=\mathrm{A} 4$ 52SK1\&search Type $=$ RAPID\&matchedCatNo= A452SK1;

https://www.fishersci.com/shop/products/tetrah ydrofuran-hplc-fisher-chemical-

5/t425sk1 ? searchHijack $=$ true \&searchTerm $=\mathrm{T} 42$

$5 \mathrm{SK} 1 \&$ search $\mathrm{Type}=\mathrm{RAPID} \&$ matchedCatNo $=\mathrm{T}$ 425SK 1 ;

https://www.fishersci.com/shop/products/2-

propanol-hplc-fisher-chemical-

5/a451sk1 searchHijack $=$ true \&search Term $=\mathrm{A} 4$ 51SK1\&search Type $=$ RAPID\&matchedCatNo= A451SK1 (accessed 28 January 2018).

Ponomarev Phillip V. - postgraduate student, Lab of Sorption Methods, GEOKHI RAS, Moscow, e-mail: filpon@mail.ru

Dolgonosov Anatoly M. - Dr.Sci.(Chem.), Leading scientific associate, Lab of Sorption Methods, GEOKHI RAS, Prof. of Chemistry Chair, University «Dubna», Moscow , e-mail: amdolgo@mail.ru 